\title{
The role of renin angiotensin system antagonists in the prevention of doxorubicin and trastuzumab induced cardiotoxicity
}

Gauri Akolkar', Navdeep Bhullar ${ }^{1}$, Hilary Bews ${ }^{1}$, Bilal Shaikh', Sheena Premecz ${ }^{1}$, Kimberly-Ann Bordun ${ }^{1}$, David YC Cheung ${ }^{1}$, Vineet Goyal ${ }^{1}$, Anita K Sharma ${ }^{1}$, Philip Garber ${ }^{2}$, Pawan K Singal ${ }^{1}$ and Davinder S Jassal $\left.\right|^{1,2,3,4,5^{*}}$

\begin{abstract}
Background: Cardio-Oncology is an evolving discipline that focuses on the management of cancer patients who develop cardiovascular complications as a result of their treatment. Although the current combination of surgical resection, radiation, and chemotherapy may lead to a cure in cancer patients, the administration of anti-cancer drugs, in particular Doxorubicin (DOX) and Trastuzumab (TRZ), is associated with an increased risk of cardiotoxicity. Little is known on the potential cardioprotective role of renin angiotensin system (RAS) antagonists in the prevention of DOX+TRZ mediated cardiotoxicity.
\end{abstract}

Objective: The aim of the study was to determine whether RAS antagonists would be useful in attenuating DOX+TRZ induced cardiotoxicity.

Methods: A total of 240 C57BI/6 mice were randomized to prophylactic treatment with placebo, Aliskiren, Perindopril, or Valsartan for a total of 13 weeks. Within each arm, mice received treatment with either DOX, TRZ, or the combination of both drugs. Serial murine echocardiography was performed weekly to characterize the degree of cardiovascular remodeling within each group.

Results: In wild-type (WT) mice treated with DOX+TRZ, LV end diastolic internal diameter (LVID) increased from $3.1 \pm 0.2 \mathrm{~mm}$ at baseline to $4.6 \pm 0.3 \mathrm{~mm}$ at week $13(p<0.05)$ and the LV fractional shortening (FS) decreased from $52 \pm 2 \%$ at baseline to $26 \pm 2 \%$ at week $13(p<0.05)$. Prophylactic treatment with Aliskiren, Perindopril, or Valsartan attenuated the degree of LV cavity dilatation with LVID dimensions of $3.9 \pm 0.2 \mathrm{~mm}, 4.1 \pm 0.2 \mathrm{~mm}$, and $4.2 \pm 0.1 \mathrm{~mm}$ at week 13 , respectively $(p<0.05)$. Similarly, prophylactic treatment with Aliskiren, Perindopril, or Valsartan was partially cardioprotective with FS of $40 \pm 1 \%, 32 \pm 1 \%$, and $33 \pm 2 \%$ at week 13 , respectively $(p<0.05)$. As compared to WT mice receiving DOX+TRZ, prophylactic treatment with RAS inhibition was also associated with improved survival, corroborating the echocardiographic findings.

Conclusion: The cardiotoxic effects of DOX+TRZ were partially attenuated by the prophylactic administration of RAS antagonists in a chronic murine model of chemotherapy induced cardiac dysfunction.

Keywords: Cardio-Oncology, Doxorubicin, Trastuzumab, RAS antagonists, Murine echocardiography

\footnotetext{
* Correspondence: djassal@sbgh.mb.ca

${ }^{1}$ Institute of Cardiovascular Sciences, St. Boniface Research Centre, University

of Manitoba, Winnipeg, Manitoba, Canada

${ }^{2}$ Section of Cardiology, Department of Internal Medicine, University of

Manitoba, Winnipeg, Manitoba, Canada

Full list of author information is available at the end of the article
} 


\section{Introduction}

As the second leading cause of cancer related deaths in women, breast cancer constitutes a major health concern. In Canada alone, approximately 22,000 women are diagnosed with breast cancer each year and over 5,000 die from the disease [1,2]. Anthracyclines, including Doxorubicin (DOX), are a class of chemotherapeutic agents with demonstrated efficacy in the breast cancer setting [3-6]. The clinical utility of DOX is limited, however, by its inherent cardiotoxicity. The average incidence of DOX induced heart failure is $5 \%$ at a cumulative dose of $400 \mathrm{mg} / \mathrm{m}^{2}$ and increases to $25 \%$ with cumulative doses above $550 \mathrm{mg} / \mathrm{m}^{2}$ [3-6]. In addition to DOX, Trastuzumab (TRZ), a monoclonal antibody against the extracellular domain of the human epidermal growth factor receptor 2 protein (HER2), is used in both the adjuvant and metastatic settings of HER2 positive breast cancer [7-12]. The addition of TRZ to adjuvant chemotherapy in patients with HER2 positive breast cancer was unequivocally beneficial, facilitating a 50\% decrease in the risk of cancer relapse and a 33\% decrease in mortality [7-12]. Despite its widespread adoption, however, it is now recognized that TRZ potentiates the cardiotoxic side effects of DOX. Although clinical trials have reported the risk of developing LV systolic dysfunction following DOX+TRZ treatment to be as great as $10 \%$ [9-11], recent real world studies have indicated that nearly 1 in 4 women will develop a drug induced cardiomyopathy, demonstrating the clinical significance of this disease [13-17].

Among the potential mechanisms for DOX+TRZ mediated cardiotoxicity, activation of the renin-angiotensin system (RAS) has gained recent attention. In an animal model of chemotherapy induced cardiomyopathy, chronic DOX administration ( $1 \mathrm{mg} / \mathrm{kg}$ biweekly, for 8 weeks) was associated with a 2 -fold increase in plasma renin activity, the enzyme responsible for initiating the RAS [18]. In a separate study, cardiac neurohumoral activation, signified by increased expression of pro-atrial naturetic peptide (ANP) mRNA, was observed in rabbits treated with $2 \mathrm{mg} / \mathrm{kg}$ DOX [19]. As RAS activation was shown to trigger ANP expression, these findings suggested that cardiac neurohumoral activation was mediated through an increase in the RAS [20]. Finally, in a chronic rat model, a cumulative dose of $420 \mathrm{mg} / \mathrm{kg}$ DOX facilitated up-regulation of the angiotensin II type 1 receptor $\left(\mathrm{AT} 1_{\mathrm{R}}\right)$ [21]. Additional studies have demonstrated the downstream cardiotoxic effects of $A T 1_{R}$ signalling, including stimulation of $\mathrm{NADPH}$ oxidase to generate oxidative stress, and activation of mitogen-activated protein kinases (MAPKs), responsible for cellular growth, inflammation, hypertrophy, and apoptosis [22,23].

RAS involvement in the pathophysiology of DOX+TRZ mediated cardiac dysfunction has raised the question as to whether the prophylactic use of RAS antagonists could potentially mitigate these cardiotoxic effects. Previous basic science studies have demonstrated that the prophylactic administration of angiotensin converting enzyme inhibition (ACEI), including Captopril, Enalapril, and Lisinopril, was partially cardioprotective in both acute and chronic animal models of DOX induced cardiomyopathy $[19,24,25]$. In a rabbit model of DOX mediated cardiomyopathy, $1 \mathrm{mg} / \mathrm{kg} /$ day oral Lisinopril for a total of 10 weeks attenuated cardiomyocyte loss and ANP mRNA expression, in comparison to rabbits receiving DOX alone [19]. Furthermore, intragastric administration of Captopril $(10 \mathrm{mg} / \mathrm{kg})$ or Enalapril $(2 \mathrm{mg} / \mathrm{kg})$ for 7 days resulted in a decline in lipid peroxidation, and enzymatic indicators of acute cardiac toxicity in a rat model of DOX induced cardiomyopathy [24]. Little is known, however, on the utility of antagonizing the RAS pathway in a hierarchical fashion for the prevention of combined DOX+TRZ mediated cardiotoxicity.

The objective of the current study was to determine whether inhibition of the RAS pathway at three distinct levels, including direct renin inhibition (DRI; Aliskiren), angiotensin converting enzyme inhibition (ACEI; Perindopril), and angiotensin receptor blockade (ARB; Valsartan) can prevent cardiac dysfunction in a chronic in vivo model of DOX+TRZ mediated cardiotoxicity.

\section{Methods}

\section{Experimental animals}

Animal procedures were conducted in accordance with guidelines published by the Canadian Council on Animal Care. All procedures, including drug administration and longitudinal echocardiographic studies, were approved by the Animal Protocol Review Committee at the University of Manitoba.

Adult male C57Bl/6 mice, weighing $20-25$ g, were obtained from Jackson Laboratories (MA, US). The animals were housed under controlled environmental conditions, including temperature, humidity, and lighting. Standard laboratory chow and water were provided ad libitum.

\section{Experimental protocol}

A total of $240 \mathrm{C} 57 \mathrm{Bl} / 6$ male mice were randomized to one of the following prophylactic treatment arms: (A) placebo (saline; $n=60)$; (B) Aliskiren ( $50 \mathrm{mg} / \mathrm{kg} ; \mathrm{n}=60$ ); (C) Perindopril (3 $\mathrm{mg} / \mathrm{kg} ; \mathrm{n}=60)$; or (D) Valsartan $(10 \mathrm{mg} / \mathrm{kg} ; \mathrm{n}=60)$ (Figure 1). RAS antagonists were administered orally by gavage on a daily basis for the entire study period of 13 weeks. Furthermore, mice from each prophylactic treatment arm were randomized to one of the following chemotherapeutic regimens: (i) TRZ (4 mg/kg weekly, intraperitoneal (i.p.); $\mathrm{n}=20)$; (ii) DOX (4 mg/kg weekly, i.p.; $\mathrm{n}=20$ ); or (iii) DOX+TRZ $(\mathrm{n}=20)$ (Figure 1). TRZ, DOX, or DOX+TRZ injections were initiated at week 2 , following 2 weeks of prophylactic treatment with a 


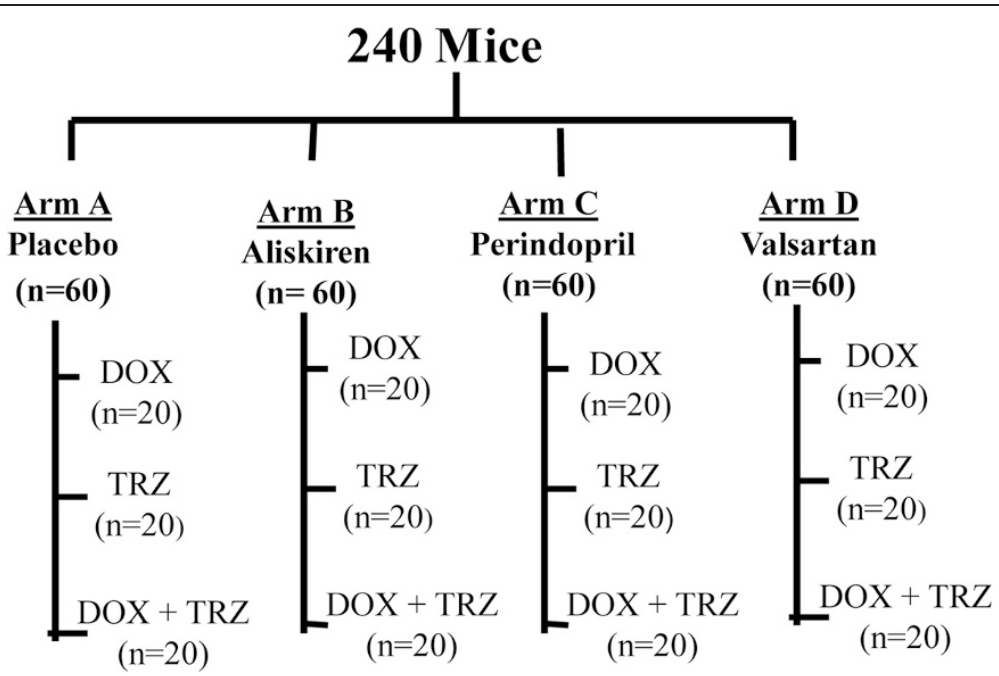

Figure $1 \mathrm{~A}$ total of $240 \mathrm{C} 57 \mathrm{BI} / 6$ mice were randomized to one of the following prophylactic treatment arms: (A) placebo (saline; $n=60$ ); (B) Aliskiren (50 mg/kg; $\mathrm{n}=60)$; (C) Perindopril ( $3 \mathrm{mg} / \mathrm{kg} ; \mathrm{n}=60)$; or (D) Valsartan (10 mg/kg; $\mathrm{n}=60)$. RAS antagonists were administered orally on a daily basis for the entire study period of 13 weeks. Furthermore, mice from each prophylactic treatment arm were randomized to one of the following chemotherapeutic regimens: (i) TRZ (4 mg/kg weekly, intraperitoneal (i.p.); $\mathrm{n}=20$ ); (ii) DOX (4 mg/kg weekly, i.p.; $\mathrm{n}=20$ ); or (iii) DOX+TRZ $(n=20)$.

RAS antagonist or placebo, and continued for 5 weeks (Figure 2). The cumulative doses of DOX or TRZ achieved were the minimum concentration to induce a chemotherapy mediated cardiomyopathy, as previously validated by our group and others [26-28]. Cardiac function was evaluated over the course of the study via serial murine echocardiography. Mice were imaged at baseline and weekly until euthanization at week 13 .

\section{Murine echocardiography}

In vivo cardiac function was evaluated by serial murine echocardiography, as previously described [27]. Awake mice were imaged at baseline and weekly thereafter, for the duration of the 13-week study (Figure 2). Images were captured in $\mathrm{M}$-mode and the $2 \mathrm{D}$ parasternal short axis view, using a $13-\mathrm{MHz}$ linear array ultrasound probe (Vivid 7, GE Medical Systems, Milwaukee, WI, USA). $\mathrm{M}$-mode recordings were used to evaluate indices of cardiac dimension and function: LV end diastolic internal diameter (LVID), LV end systolic internal diameter (LVIS), interventricular septal thickness (IVS), posterior wall thickness (PWT), LV fractional shortening (LVFS), and LV ejection fraction (LVEF). Echocardiographic images were processed offline using EchoPAC v110.0.0 PC

\section{Echocardiography}

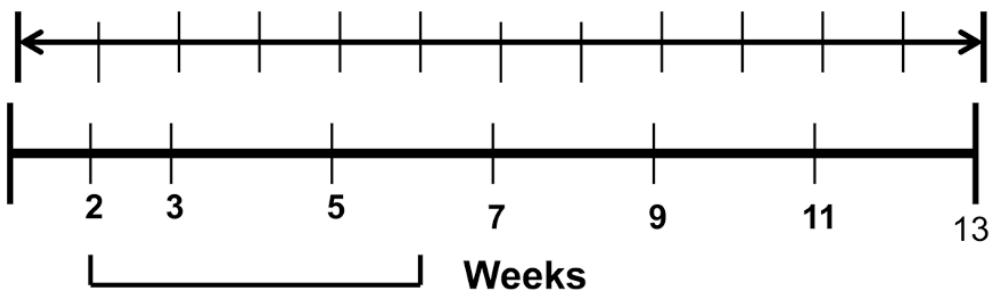

Anti-Cancer Drugs

DOX, TRZ and DOX+TRZ

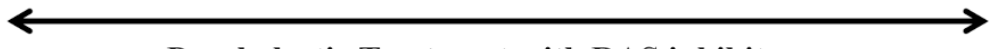

Prophylactic Treatment with RAS inhibitors Aliskiren, Perindopril and Valsartan

Figure 2 Timeline for drugs administered to mice in each group. Mice received prophylactic treatment with Aliskiren (50 mg/kg), Perindopril (3 mg/kg) or Valsartan $(10 \mathrm{mg} / \mathrm{kg}$ ) in drinking water daily. Prophylactic treatment was started 2 weeks prior to chemotherapy with DOX, TRZ and DOX + TRZ and was continued for 13 weeks. DOX (4 mg/kg), TRZ (4 mg/ $\mathrm{kg})$ and DOX + TRZ (4 mg/kg) was administered by weekly intraperitoneal injection for a total of 5 weeks. Cardiac function was monitored by echocardiography on a weekly basis for 13 weeks. 
software (Vivid 7, GE Medical Systems, Milwaukee, WI, US).

A total of 40 mice, from various treatment groups, were randomly selected in order to assess the variability associated with echocardiographic measurements of LV cavity dimensions and function. To determine intraobserver variability, repeat LVID and LVFS measurements were made by a single observer (DJ), two weeks apart. Inter-observer variability was established by comparing LVID and LVFS values calculated by two independent observers (NB and DJ). Intra- and inter-observer variability were reported as the absolute value of the difference between the two observations divided by the mean.

\section{Statistical analysis}

Statistical analysis was conducted using SPSS 15.0 and GraphPad Prism 5, with echocardiographic data expressed as a mean $\pm \mathrm{SD}$. An analysis of variance approach for repeated measurements comparison of echocardiographic parameters over time was used. One-way ANOVA and post-hoc Dunnett's tests were performed when necessary. A P value $<0.05$ was considered statistically significant.

\section{Results}

\section{Survival}

The overall survival of C57Bl/6 mice administered TRZ, DOX, or DOX+TRZ, with or without RAS inhibitor prophylaxis is represented in Figure 3. In mice treated with TRZ alone, the survival rate was $100 \%$. The administration of either DOX alone or the combination of DOX+TRZ resulted in survival rates to be significantly reduced to $50 \%$ and $30 \%$, respectively, by the end of the study. Conversely, prophylactic administration of a RAS inhibitor attenuated the increased mortality rates observed with both DOX and DOX+TRZ therapy. For mice in the DOX treatment arm, overall survival was significantly increased when pretreated with Aliskiren, Perindopril, or Valsartan $(\mathrm{p}<0.05)$. Similarly, overall survival following DOX+TRZ treatment

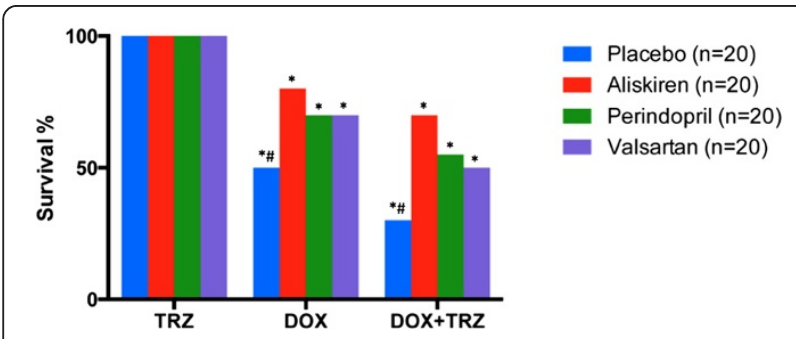

Figure 3 Survival probability at the end of 13 weeks for C57BI/6 mice receiving prophylactic treatment with RAS inhibitors Aliskiren, Perindopril or Valsartan and then treated with anti-cancer drugs TRZ, DOX or the combination of DOX+TRZ. ${ }^{*} p<0.05$ significant as compared to TRZ. ${ }^{*} p<0.05$ comparing placebo to either Aliskiren, Perindopril, or Valsartan. was significantly increased by the prophylactic administration of one of the RAS antagonists (Figure 3).

\section{Echocardiographic indices}

Echocardiographic indices, including HR, PWT, LVID, LVFS, and LVEF were within normal physiologic limits for all mice at baseline. No significant change from baseline value was observed for $\mathrm{HR}$ or PWT at week 13 in any of the treatment arms (Table 1). In C57Bl/6 mice treated with TRZ, there were no significant changes in LV cavity dimensions as compared to baseline (Figure 4A). Mice treated with DOX alone demonstrated a significant increase in cavity dimensions, as the LVID increased from $3.2 \pm 0.1 \mathrm{~mm}$ at baseline to $4.5 \pm 0.2 \mathrm{~mm}$ at week $13(\mathrm{p}<0.05$, Figure $4 \mathrm{~B})$. Mice receiving DOX+TRZ treatment demonstrated a similar increase in LVID from $3.1 \pm 0.2 \mathrm{~mm}$ at baseline to $4.6 \pm 0.3 \mathrm{~mm}$ at week $13(\mathrm{p}<0.05$, Figure $4 \mathrm{C})$. Pretreatment with Aliskiren, Perindopril, and Valsartan significantly reduced LVID in mice administered DOX alone from $4.5 \pm 0.2 \mathrm{~mm}$ to $3.6 \pm 0.2 \mathrm{~mm}, 3.9 \pm 0.2 \mathrm{~mm}$, and $4.0 \pm 0.2 \mathrm{~mm}$, respectively, at week $13(\mathrm{p}<0.05$; Figure 4B). Similarly, in mice treated with DOX+TRZ, the prophylactic administration of Aliskiren, Perindopril, and Valsartan significantly reduced LVID at week 13 from $4.6 \pm 0.3 \mathrm{~mm}$ to $3.9 \pm 0.2 \mathrm{~mm}, 4.1 \pm 0.2 \mathrm{~mm}$, and $4.2 \pm 0.1 \mathrm{~mm}$, respectively, $(\mathrm{p}<0.05$; Figure $4 \mathrm{C})$.

LVFS did not change from baseline values in mice administered TRZ over the course of the 13 week study (Figure 5A). In mice treated with DOX alone, LVFS decreased from $51 \pm 1 \%$ at baseline to $32 \pm 2 \%$ at week 13 $(\mathrm{p}<0.05$; Figure $5 \mathrm{~B})$. Pre-treatment with Aliskiren, Perindopril, and Valsartan attenuated DOX induced LV impairment, improving LVFS values to $45 \pm 1 \%, 38 \pm 1 \%$, and $37 \pm 2 \%$, respectively, at week $13(\mathrm{p}<0.05$; Figure $5 \mathrm{~B})$. In mice treated with the combination of DOX+TRZ, LVFS further decreased from $52 \pm 2 \%$ at baseline to $26 \pm 2 \%$ at week $13(\mathrm{p}<0.05$; Figure 5C). Prophylactic treatment with Aliskiren, Perindopril, and Valsartan significantly improved LVFS to $40 \pm 1 \%, 32 \pm 1 \%$, and $33 \pm 2 \%$, respectively, at week 13 ( $\mathrm{p}<0.05$; Figure 5 C). The LVEF values corroborated the LVFS findings as shown in Figure 6.

Intra- and inter-observer variabilities of LVID and LVFS parameters are summarized in Table 2. Overall, there was good to excellent intra- and inter-observer variabilities for LVID and LVFS measurements.

\section{Discussion}

In Canada, breast cancer is the most common female malignancy and is the second leading cause of cancer related deaths in women. Despite the widespread use of DOX+TRZ in breast cancer treatment, efficacy is limited by the inherent cardiotoxicity of this regimen. Consequently, cardiac dysfunction has been identified in approximately 1 in 4 women receiving this anti- 
Table 1 Echocardiographic parameters

\begin{tabular}{|c|c|c|c|c|c|}
\hline Echo variable & Treatment arm & Group & Baseline & Week 13 & $\mathbf{p}$ \\
\hline & & TRZ & $717 \pm 12$ & $729 \pm 10$ & 0.62 \\
\hline & Placebo & DOX & $725 \pm 10$ & $701 \pm 18$ & 0.58 \\
\hline & & DOX+ TRZ & $690 \pm 14$ & $695 \pm 18$ & 0.82 \\
\hline \multirow[t]{14}{*}{ Heart Rate (bpm) } & & TRZ & $735 \pm 15$ & $740 \pm 12$ & 0.88 \\
\hline & Aliskiren & DOX & $705 \pm 14$ & $701 \pm 21$ & 0.72 \\
\hline & & DOX+ TRZ & $705 \pm 14$ & $701 \pm 21$ & 0.72 \\
\hline & & TRZ & $705 \pm 18$ & $710 \pm 12$ & 0.9 \\
\hline & Perindopril & DOX & $722 \pm 14$ & $720 \pm 18$ & 0.77 \\
\hline & & DOX+ TRZ & $722 \pm 14$ & $720 \pm 18$ & 0.77 \\
\hline & & TRZ & $744 \pm 13$ & $735 \pm 12$ & 0.67 \\
\hline & Valsartan & DOX & $695 \pm 14$ & $705 \pm 12$ & 0.72 \\
\hline & & DOX + TRZ & $695 \pm 14$ & $705 \pm 12$ & 0.72 \\
\hline & & TRZ & $0.82 \pm 0.02$ & $0.84 \pm 0.01$ & 0.92 \\
\hline & Placebo & DOX & $0.83 \pm 0.02$ & $0.81 \pm 0.01$ & 0.97 \\
\hline & & $\mathrm{DOX}+\mathrm{TRZ}$ & $0.84 \pm 0.01$ & $0.83 \pm 0.01$ & 0.76 \\
\hline & & TRZ & $0.80 \pm 0.03$ & $0.83 \pm 0.02$ & 0.52 \\
\hline & Aliskiren & DOX & $0.83 \pm 0.02$ & $0.81 \pm 0.02$ & 0.68 \\
\hline \multirow[t]{7}{*}{ PWT (mm) } & & DOX + TRZ & $0.80 \pm 0.04$ & $0.82 \pm 0.02$ & 0.82 \\
\hline & & TRZ & $0.81 \pm 0.04$ & $0.82 \pm 0.02$ & 0.82 \\
\hline & Perindopril & DOX & $0.83 \pm 0.02$ & $0.81 \pm 0.02$ & 0.58 \\
\hline & & $\mathrm{DOX}+\mathrm{TRZ}$ & $0.82 \pm 0.04$ & $0.83 \pm 0.01$ & 0.76 \\
\hline & & TRZ & $0.82 \pm 0.01$ & $0.84 \pm 0.01$ & 0.67 \\
\hline & Valsartan & DOX & $0.82 \pm 0.03$ & $0.83 \pm 0.02$ & 0.72 \\
\hline & & DOX+ TRZ & $0.84 \pm 0.02$ & $0.83 \pm 0.01$ & 0.76 \\
\hline
\end{tabular}

PWT, posterior wall thickness.

cancer therapy [13-17]. Despite an increased understanding of the potential mechanisms contributing to DOX + TRZ mediated cardiotoxicity, there is currently no approved prophylactic therapy to prevent chemotherapy induced cardiomyopathy in breast cancer patients. In the current study, we demonstrated that the cardiotoxic side effects of DOX+TRZ are partially attenuated by the prophylactic administration of RAS antagonists, as characterized by an improvement in overall survival rate and prevention of adverse cardiovascular remodeling.

Several previous studies using murine models have illustrated the significant mortality associated with DOX administration. Neilan et al. demonstrated a survival rate of $35 \%$ at week 16 in $\mathrm{C} 57 \mathrm{Bl} / 6$ mice receiving $\mathrm{DOX}$ (4 $\mathrm{mg} / \mathrm{kg}$ weekly) for 5 weeks [29]. Similarly, in rats treated with DOX (4 mg/kg weekly) for 6 weeks, a decreased survival rate of $75 \%$ was reported at the end of a 10 week study [25]. This present study demonstrated similar reductions in overall survival (50\%) for mice in the DOX treatment arm at 13 weeks. The effect was even more pronounced in animals receiving combination therapy with
DOX+TRZ. Conversely, prophylactic treatment with RAS inhibitors provided survival benefits. These basic science results provide encouraging evidence for the potential use of RAS antagonists in the prevention of DOX+TRZ mediated cardiomyopathy, which warrants further study in the clinical setting.

In addition to the survival benefits conferred by the prophylactic administration of RAS inhibitors, there is an improvement in adverse cardiovascular remodeling in our chronic DOX+TRZ mediated cardiotoxicity model. Anthracyclines, including DOX, affect hemodynamic and echocardiographic parameters, leading to depressed cardiac function in murine models of chemotherapeutic regimens [26-29]. In an acute murine model of chemotherapy mediated cardiotoxicity, we previously demonstrated severe LV systolic dysfunction due to DOX treatment, as exemplified by LV cavity dilatation and reduced LVEF [26]. The effect was even more pronounced in animals receiving the combination of DOX+TRZ, as the LVFS decreased by $14 \%$ and the EF decreased by $20 \%$ at day 10 , as compared to baseline [26]. Similar results were obtained by Neilan et al. in C57Bl/6 mice treated with a single dose 


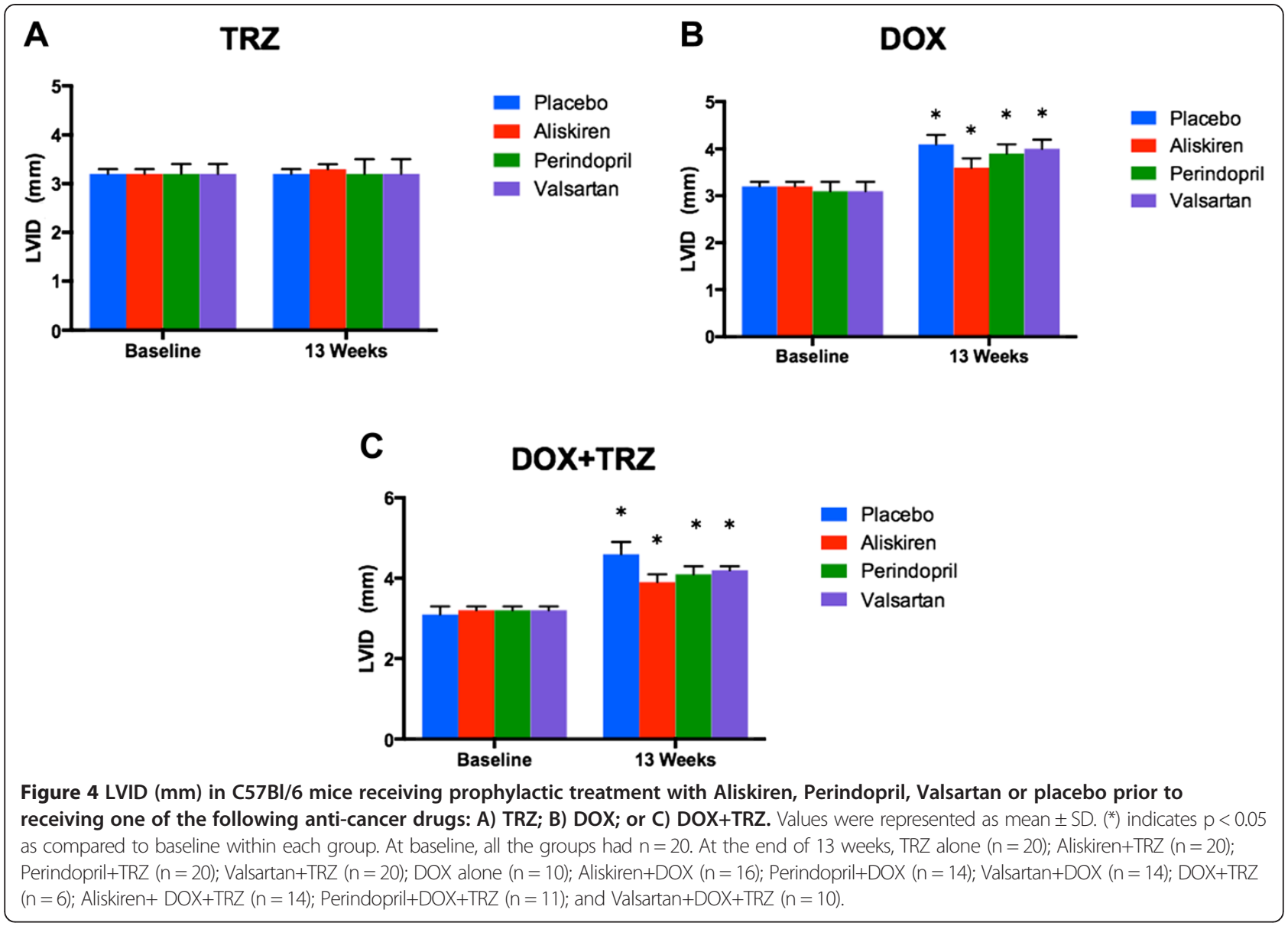

of $20 \mathrm{mg} / \mathrm{kg}$ DOX [29]. In their study, LVFS and EF decreased by $17 \pm 1 \%$ and $13 \pm 1 \%$ respectively, by day 5 [29]. A chronic study of DOX administration $(4 \mathrm{mg} / \mathrm{kg}$ for 5 weeks) performed by the same group, further demonstrated a reduction in LVFS and EF by $29 \%$ and $36 \%$, respectively from baseline value at 16 weeks [29]. Our current study adds to the existing literature by confirming the cardiotoxic effects of DOX in a chronic murine model. Specifically, we demonstrated LV systolic dysfunction, as illustrated by a reduction in LVFS and LVEF and concurrent increase in LVID. Furthermore, we demonstrated the increased cardiotoxic side effect in mice administered the combination of DOX+TRZ. In accordance with the aforementioned mortality benefits, RAS inhibition mitigated the adverse cardiac remodelling associated with DOX+TRZ treatment.

Although the pathogenesis of DOX+TRZ mediated cardiotoxicity is multifactorial, there is accumulating evidence to suggest the RAS as a key contributor. Angiotensin II plays an important role as a vasoconstrictor agent and a mitogenic factor by interacting with the $\mathrm{AT} 1_{\mathrm{R}}$ in cardiovascular myocytes [30]. Although DOX induces myofibrillar loss, apoptosis, and significantly impairs contractile function in WT mice, this drug induced cardiotoxicity was not observed in $\mathrm{AT} 1_{\mathrm{R}}$-knockout mice or in animals treated with $\mathrm{AT} 1_{\mathrm{R}}$ blockade [30]. These findings suggest a contributory role for the RAS pathway in the development of DOX mediated cardiotoxicity. The administration of the ARB Telmisartan (10 mg/kg/day for 7 days), was cardioprotective in rats treated with a single dose of $20 \mathrm{mg} / \mathrm{kg}$ DOX, by decreasing lipid peroxidation, GSH depletion, and oxidative stress [31]. Additionally, in a chronic rat model of DOX induced cardiotoxicity (DOX $25 \mathrm{mg} / \mathrm{kg}$ for a 6 week period), Enalapril served to preserve mitochondrial function, down-regulate free radical production, and preserve overall cardiac function [25]. Finally, in a chronic rat model of DOX $(15 \mathrm{mg} / \mathrm{kg})$ mediated cardiac dysfunction, the prophylactic administration of either ACEI (Captopril $60 \mathrm{mg} / \mathrm{kg}$ ) or ARB (Telmisartan $10 \mathrm{mg} / \mathrm{kg}$ ) proved to be cardioprotective [32]. This was evidenced by a significant reduction in the concentration of cardiac biomarkers and oxidative stress, and by the maintenance of characteristic cardiac histology in rats pre-treated with RAS antagonism. Our current study adds to the existing literature which supports the cardioprotective capabilities of RAS antagonism in a chronic murine model of chemotherapy induced cardiac dysfunction. Our study is the first to also demonstrate the potential cardioprotective role of 

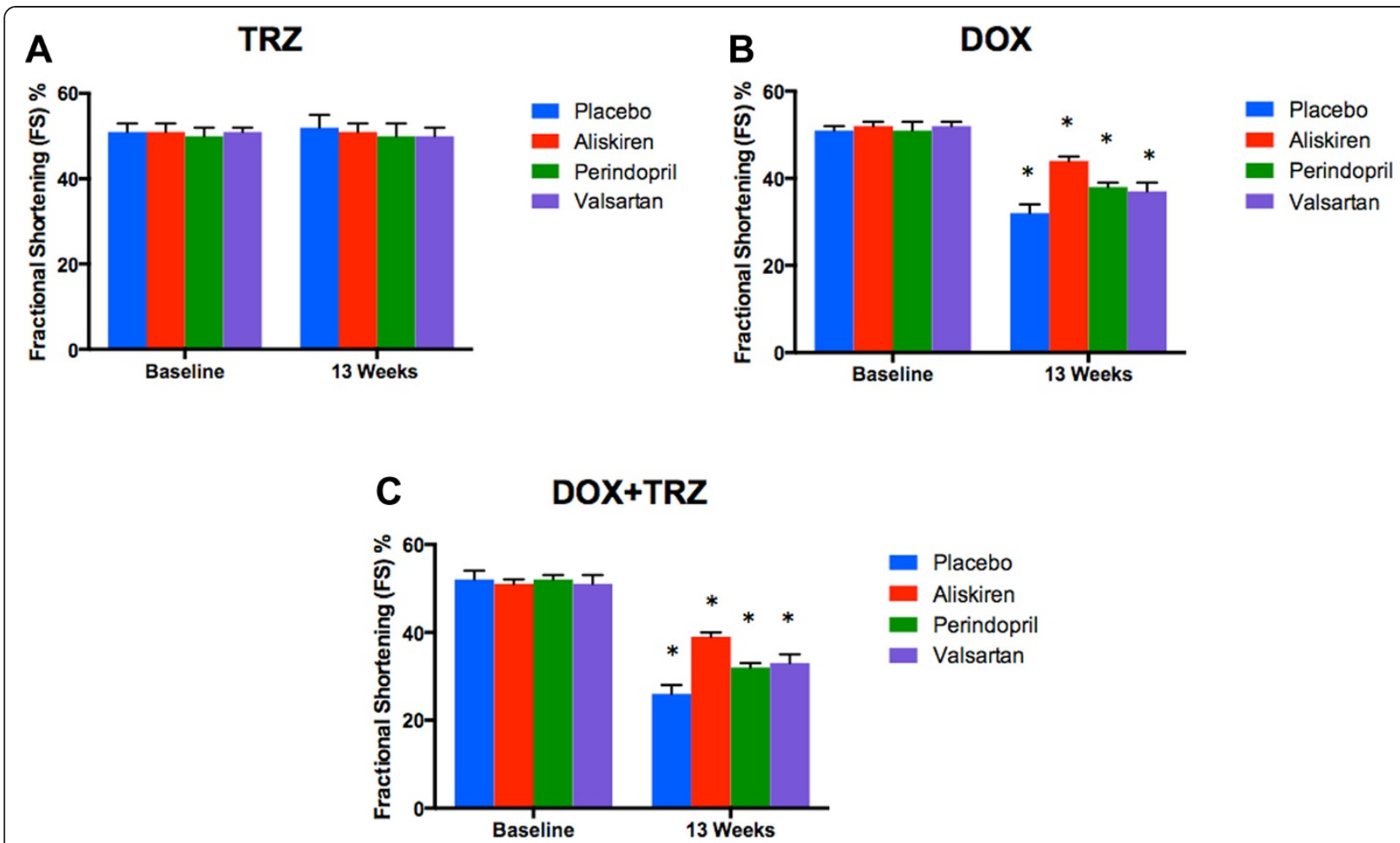

Figure 5 Fractional shortening of C57BI/6 mice receiving prophylactic treatment with Aliskiren, Perindopril, Valsartan or placebo prior to receiving one of the following anti-cancer drugs: A) TRZ; B) DOX; or C) DOX+TRZ. Values were represented as mean \pm SD. (*) indicates $p<0.05$ as compared to baseline within each group. At baseline, all the groups had $n=20$. At the end of 13 weeks, TRZ alone $(n=20)$; Aliskiren+TRZ ( $n=20)$; Perindopril+TRZ $(n=20)$; Valsartan+TRZ $(n=20)$; DOX alone $(n=10)$; Aliskiren+DOX $(n=16)$; Perindopril+DOX $(n=14)$; Valsartan+DOX $(n=14)$; DOX+TRZ $(n=6)$; Aliskiren+DOX+TRZ $(n=14)$; Perindopril+DOX+TRZ $(n=11)$; and Valsartan+DOX+TRZ $(n=10)$.

direct renin inhibition, specifically Aliskiren, in preventing DOX mediated cardiotoxicity. Additionally, although previous animal studies have focussed on a DOX only model $[19,24,25,31,32]$, we have extended our important findings to a combined DOX+TRZ model, mimicking the clinical setting of breast cancer.

Translating these basic science findings to the clinical arena, a number of clinical studies have evaluated the potential cardioprotective role of RAS antagonists in the clinical setting of chemotherapy mediated cardiotoxicity. Although the administration of ACEI or ARB is associated with reduced morbidity and mortality in the treatment of heart failure [33], a limited number of clinical studies have demonstrated the potential benefits of RAS inhibition in the prevention of DOX mediated cardiotoxicity. In a small prospective study including 49 patients with various cancers responsive to an anthracycline based chemotherapy regimen, Dessi et al. demonstrated that ARB was able to reverse acute myocardial dysfunction up to 12 months of follow-up [34]. Cardinale at al. demonstrated that ACEI can prevent a decline in LVEF and cardiac events in cancer patients receiving high dose anthracyclines [35]. In a recent meta-analysis, the prophylactic administration of ACEI or ARB in patients receiving an anthracycline based regimen was associated with a relative risk of 0.11 for the development of cardiotoxicity compared to placebo [36]. The recent OVERCOME trial involved 90 patients with malignant hemopathies, randomized to receive prophylactic ACEI (Enalapril) and betablockers (Carvedilol) at least 24 hours prior to chemotherapy or to a control group [37]. The results suggested that combination pre-treatment with Enalapril and Carvedilol was cardioprotective, as a lower incidence of death, heart failure and LV systolic dysfunction was observed in the intervention group. The ongoing MANTICORE study is similarly investigating the cardioprotective role of angiotensin converting enzyme inhibition and beta-blockers in HER2 positive breast cancer patients treated with TRZ [38]. Patients were randomized to prophylactic ACEI (Perindopril), beta-blocker (Bisoprolol) or placebo, 7 days prior to TRZ therapy. The MANTICORE study will determine if DOX+TRZ mediated remodelling and systolic dysfunction is attenuated by the prophylactic administration of ACEI and beta-blockers in the clinical setting of breast cancer. In our current study, as the cardiotoxic side effects of DOX+TRZ are partially attenuated by the prophylactic administration of RAS antagonists, in particular by DRI as compared to either ACEI or ARB, future clinical 


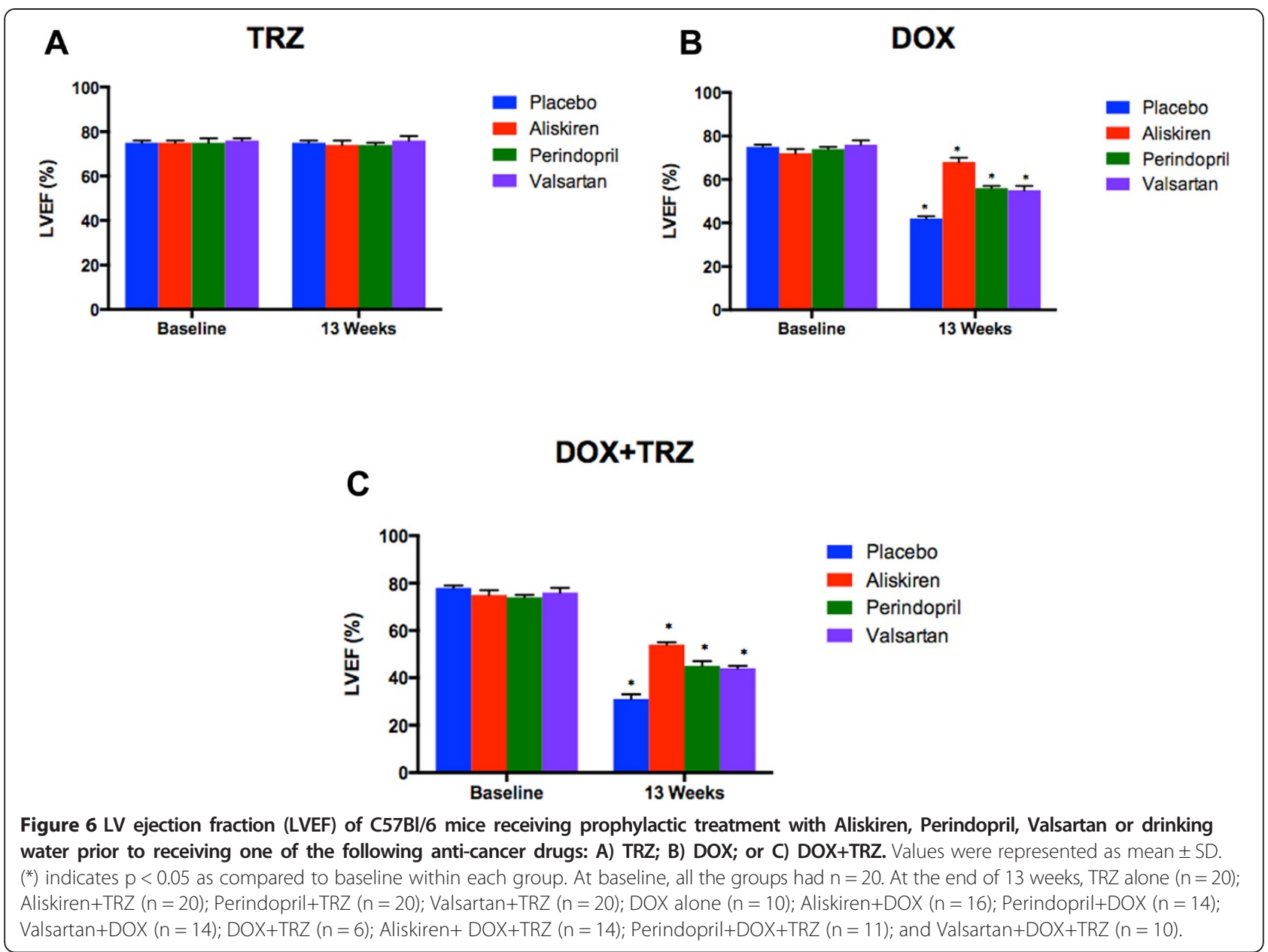

studies evaluating the cardioprotective role of Aliskiren in the breast cancer setting are warranted.

There were some limitations to our study. First, although both DOX+TRZ were co-administered on a weekly basis to produce a chronic murine model of chemotherapy induced cardiotoxicity, adjuvant therapy with TRZ should ideally be given after treatment with an anthracycline based chemotherapy regimen to mimic the clinical setting of breast cancer. Second, although Aliskiren

Table 2 Intra- and inter-observer variabilities of the echocardiographic parameters

\begin{tabular}{ll}
\hline Echocardiographic parameters & Mean difference \pm SD \\
\hline Intra-observer variability & \\
LVID (mm) & $0.05 \pm 0.02$ \\
LVFS (\%) & $0.6 \pm 0.2$ \\
Inter-observer variability & \\
LVID (mm) & $0.08 \pm 0.04$ \\
LVFS (\%) & $1.3 \pm 0.2$ \\
\hline
\end{tabular}

LVID, Left ventricular end diastolic internal diameter; LVFS, Left ventricular fractional shortening. is commonly used as a primary treatment agent for hypertension, recent randomized controlled trials, including the ASTRONAUT study, have failed to demonstrate a benefit of DRI in reducing overall morbidity and mortality in the heart failure setting [39]. In the current study, we demonstrated that Aliskiren may be more cardioprotective as compared to ACEI and ARB, suggesting a need for a clinical study to further evaluate this hypothesis generating finding in the Cardio-Oncology population. Finally, future mechanistic studies focusing on oxidative stress and apoptosis are required to understand the potential cardiprotective effects of the various RAS antagonists in the setting of DOX+TRZ mediated cardiotoxicity.

\section{Conclusions}

The cardiotoxic effects of DOX+TRZ were partially attenuated by the prophylactic administration of RAS antagonists in a chronic murine model of chemotherapy induced cardiac dysfunction. 


\section{Authors' contributions}

GA, NB, SP, AS, PS, and DJ contributed to the experimental studies. All authors contributed to the writing of the manuscript. All authors read and approved the final manuscript.

\section{Acknowledgements}

This work was supported a grant from the Manitoba Health Research Council and the Canadian Breast Cancer Foundation.

\section{Author details}

${ }^{1}$ Institute of Cardiovascular Sciences, St. Boniface Research Centre, University of Manitoba, Winnipeg, Manitoba, Canada. ${ }^{2}$ Section of Cardiology, Department of Internal Medicine, University of Manitoba, Winnipeg, Manitoba, Canada. ${ }^{3}$ Section of Oncology, Department of Internal Medicine, University of Manitoba, Winnipeg, Manitoba, Canada. ${ }^{4}$ Department of Radiology, St. Boniface General Hospital, University of Manitoba, Winnipeg, Manitoba, Canada. ${ }^{5}$ Associate Professor of Medicine, Radiology, and Physiology, Section of Cardiology, Department of Internal Medicine, College of Medicine, Faculty of Health Sciences, Rm Y3531, Bergen Cardiac Care Centre, St. Boniface General Hospital, 409 Tache Avenue, Winnipeg, Manitoba $\mathrm{R} 2 \mathrm{H}$ 2A6, Canada.

Received: 1 February 2015 Accepted: 17 March 2015 Published online: 03 April 2015

\section{References}

1. Verma S, Sehdev S, Joy AA. Cancer therapy disparity: unequal access to breast cancer therapeutics and drug funding in Canada. Curr Oncol. 2007:14:S3-10.

2. Canadian Cancer Society/Steering Committee on Canadian Cancer Statistics 2014; Toronto, Canada. (http://www.cancer.ca/en/cancer-information/ cancer-type/breast/statistics/?region=on).

3. Singal PK, lliskovic N. Doxorubicin-induced cardiomyopathy. N Engl J Med. 1998:339:900-5

4. Lefrak EA, Pitha J, Rosenheim S, Gottieb JA. A clinicopathologic analysis of adriamycin cardiotoxicity. Cancer. 1973:32:302-14.

5. Keefe DL. Anthracycline-induced cardiomyopathy. Semin Oncol. 2001;28:2-7

6. $\mathrm{Ng} \mathrm{R}$, Green MD. Managing cardiotoxicity in anthracycline-treated breast cancers. Expert Opin Drug Saf. 2007;6:315-21.

7. Vogel CL, Cobleigh MA, Tripathy D, Gutheil JC, Harris LN, Fehrenbacher $L$, et al. Efficacy and safety of trastuzumab as a single agent in first-line treatment of HER2-overexpressing metastaticbreast cancer. J Clin Oncol. 2002;20:719-26.

8. Olin JJ, Muss HB. New strategies for managing metastatic breast cancer. Oncology (Williston Park). 2000;14:629-41. discussion 642-4, 647-8.

9. Slamon DJ, Leyland-Jones B, Shak S, Fuchs H, Paton V, Bajamonde A, et al. Use of chemotherapy plus a monoclonal antibody against HER2 for metastatic breast cancer that overexpresses HER2. N Engl J Med. 2001;344:783-92.

10. Piccart-Gebhart MJ, Procter M, Leyland-Jones B, Goldhirsch A, Untch M, Smith I, et al. Trastuzumab after adjuvant chemotherapy in HER2-positive breast cancer. N Engl J Med. 2005;353:1659-72

11. Romond EH, Perez EA, Bryant J, Suman VJ, Geyer Jr CE, Davidson NE, et al. Trastuzumab plus adjuvant chemotherapy for operable HER2-positive breast cancer. N Engl J Med. 2005;353:1673-84.

12. Jahanzeb M. Adjuvant trastuzumab therapy for HER2-positive breast cancer. Clin Breast Cancer. 2008:8:324-33.

13. Fallah-Rad N, Walker JR, Wassef A, Lytwyn M, Bohonis S, Fang T, et al. The utility of cardiac biomarkers, tissue velocity and strain imaging, and cardiac magnetic resonance imaging in predicting early left ventricular dysfunction in patients with human epidermal growth factor receptor I-positive breast cancer treated with adjuvant trastuzumab therapy. J Am Coll Cardiol. 2011:57:2263-70

14. Guglin M, Hartlage G, Reynolds C, Chen R, Patel V. Trastuzumab-induced cardiomyopathy: not as benign as it looks? A retrospective study. J Card Fail. 2009;15:651-7.

15. McArthur HL, Chia S. Cardiotoxicity of trastuzumab in clinical practice. N Engl J Med. 2007;357:94-5.
16. Tarantini L, Feola M, Albini A, Gori S, Foglietta J, Cicoira MA, et al. Heart failure in women treated with adjuvant trastuzumab for breast cancer. G Ital Cardiol (Rome). 2012;13:54S-62

17. Wadhwa D, Fallah-Rad N, Grenier D, Krahn M, Fang T, Ahmadie R, et al. Trastuzumab mediated cardiotoxicity in the setting of adjuvant chemotherapy for breast cancer: a retrospective study. Breast Cancer Res Treat. 2009:117:257-64.

18. Arnolda L, McGrath B, Cocks M, Sumithran E, Johnston C. Adriamycin cardiomyopathy in the rabbit: an animal model of low output cardiac failure with activation of vasoconstrictor mechanisms. Cardiovasc Res. 1985:19:378-82

19. Boucek Jr RJ, Steele A, Miracle A, Atkinson J. Effects of angiotensinconverting enzyme inhibitor on delayed-onset doxorubicin-induced cardiotoxicity. Cardiovasc Toxicol. 2003;3:319-29.

20. Rockman HA, Wachhorst SP, Mao L, Ross Jr J. ANG II receptor blockade prevents ventricular hypertrophy and ANF gene expression with pressure overload in mice. Am J Physiol. 1994;266:H2468-75.

21. Zong $W N$, Yang $X H$, Chen $X M$, Huang $H J$, Zheng $H J$, Qin $X Y$, et al Regulation of angiotensin-(1-7) and angiotensin II type 1 receptor by telmisartan and losartan in adriamycin-induced rat heart failure. Acta Pharmacol Sin. 2011;32:1345-50

22. Lemarié CA, Paradis P, Schiffrin EL. New insights on signaling cascades induced by cross-talk between angiotensin II and aldosterone. J Mol Med (Berl). 2008:86:673-8.

23. Nakagami H, Takemoto M, Liao JK. NADPH oxidase-derived superoxide anion mediates angiotensin II-induced cardiac hypertrophy. J Mol Cell Cardiol. 2003;35:851-9.

24. Abd El-Aziz MA, Othman Al, Amer M, El-Missiry MA. Potential protective role of angiotensin-converting enzyme inhibitors captopril and enalapril against adriamycin-induced acute cardiac and hepatic toxicity in rats. J Appl Toxicol. 2001;21:469-73.

25. Hiona A, Lee AS, Nagendran J, Xie X, Connolly AJ, Robbins RC, et al. Pretreatment with angiotensin-converting enzyme inhibitor improves doxorubicin-induced cardiomyopathy via preservation of mitochondrial function. J Thorac Cardiovasc Surg. 2011;142:396-403.

26. Jassal DS, Han SY, Hans C, Sharma A, Fang T, Ahmadie R, et al. Utility of tissue doppler and strain rate imaging in the early detection of trastuzumab and anthracycline mediated cardiomyopathy. J Am Soc Echocardiogr. 2009:22:418-24.

27. Walker JR, Sharma A, Lytwyn M, Bohonis, Thliveris J, Singal PK, et al. The cardioprotective role of probucol against anthracycline and trastuzumab-mediated cardiotoxicity. J Am Soc Echocardiogr. 2011;24:699-705

28. Nozaki N, Shishido T, Takeishi Y, Kubota I. Modulation of doxorubicin induced cardiac dysfunction in toll-like receptor-2-knockout mice. Circulation. 2004:110:2869-74.

29. Neilan TG, Jassal DS, Perez-Sanz TM, Raher MJ, Pradhan AD, Buys ES, et al. Tissue doppler imaging predicts left ventricular dysfunction and mortality in a murine model of cardiac injury. Eur Heart J. 2006;27:1868-75.

30. Toko H, Oka T, Zou Y, Sakamoto M, Mizukami M, Sano M, et al. Angiotensin type 1a receptor mediates doxorubicin-induced cardiomyopathy. Hypertens Res. 2002;25:597-603.

31. Iqbal M, Dubey K, Anwer T, Ashish A, Pillai KK. Protective effects of telmisartan against acute doxorubicin-induced cardiotoxicity in rats. Pharmacol Rep. 2008;60:382-90.

32. Ibrahim MA, Ashour OM, Ibrahim YF, El-Bitar HI, Gomaa W, Abdel-Rahim SR. Angiotensin-converting enzyme inhibition and angiotensin AT(1)-receptor antagonism equally improve doxorubicin-induced cardiotoxicity and nephrotoxicity. Pharmacol Res. 2009;60:373-81.

33. Hunt SA. American College of Cardiology, American Heart Association Task Force on Practice Guidelines (writing committee to update the 2001 guidelines for the evaluation and management of heart failure) ACC/AHA 2005 guideline update for the diagnosis and management of chronic heart failure in the adult: a report of the American College of Cardiology/American Heart Association Task Force on practice guidelines (writing committee to update the 2001 guidelines for the evaluation and management of heart failure). J Am Coll Cardiol. 2005;46:e1-82.

34. Dessì M, Piras A, Madeddu C, Cadeddu C, Deidda M, Massa E, et al. Long-term protective effects of the angiotensin receptor blocker telmisartan on epirubicin-induced inflammation, oxidative stress and myocardial dysfunction. Exp Ther Med. 2011:2:1003-9. 
35. Cardinale D, Colombo A, Sandri MT, Lamantia G, Colombo N, Civelli M, et al. Prevention of high-dose chemotherapy-induced cardiotoxicity in high-risk patients by angiotensin-converting enzyme inhibition. Circulation. 2006;114:2474-81.

36. Kalam K, Marwick TH. Role of cardioprotective therapy for prevention of cardiotoxicity with chemotherapy: a systematic review and meta-analysis. Eur J Cancer. 2013;49:2900-9.

37. Bosch X, Rovira M, Sitges M, Domenech A, Ortiz-Perez JT, de Caralt TM, et al. Enalapril and carvedilol for preventing chemotherapy-induced left ventricular systolic dysfunction in patients with malignant hemopathies: The OVERCOME trial (prevention of left ventricular dysfunction with enalapril and carvedilol in patients submitted to intensive chemotherapy for the treatment of malignant hemopathies). J Am Coll Cardiol. 2013;61:2355-62.

38. Pituskin E, Haykowsky M, Mackey JR, Thompson RB, Ezekowitz J, Koshman S, et al. Rationale and design of the multidisciplinary approach to novel therapies in cardiology oncology research trial (MANTICORE 101-Breast): a randomized, placebo-controlled trial to determine if conventional heart failure pharmacotherapy can prevent trastuzumab-mediated left ventricular remodeling among patients with HER2+ early breast cancer using cardiac MRI. BMC Cancer. 2011;11:318.

39. Gheorghiade M, Böhm M, Greene S, Fonarow G, Lewis E, Zannad F, et al. Effect of aliskiren on postdischarge mortality and heart failure readmissions among patients hospitalized for heart failure: the ASTRONAUT randomized trial. JAMA. 2013;309:1125-35.

\section{Submit your next manuscript to BioMed Central and take full advantage of:}

- Convenient online submission

- Thorough peer review

- No space constraints or color figure charges

- Immediate publication on acceptance

- Inclusion in PubMed, CAS, Scopus and Google Scholar

- Research which is freely available for redistribution 